\title{
Corrosión e inhibición en aleaciones de aluminio de media resistencia
}

\author{
B. Davó* y J.J. de Damborenea*
}

\begin{abstract}
Resumen La elevada toxicidad de los cromatos limita cada vez más su uso en los tratamientos superficiales que se aplican a las aleaciones de aluminio en la industria aeronáutica. Por ello, es urgente encontrar inhibidores, no sólo tan eficaces como los cromatos sino, también, inocuos. En este sentido, se ha estudiado la inhibición de la corrosión de una aleación ligera de alta resistencia $\mathrm{Al}-\mathrm{Li}-\mathrm{Cu}$ (8090) en soluciones de $\mathrm{NaCl}$ con sales lantánidas de $\mathrm{CeCl}_{3}$ y $\mathrm{LaCl}_{3}$. Se ha comprobado que concentraciones comprendidas entre 100 y 10.000 ppm disminuyen la cinética de corrosión, al menos, un orden de magnitud. La superficie se modifica por la formación de una capa de óxidos e hidróxidos del catión lantánido que se ha caracterizado mediante SEM, EDS y XPS.
\end{abstract}

Palabras clave Aleaciones de aluminio. Corrosión. Inhibidores. Sales lantánidas.

\section{Corrosion and inhibition of medium-strength aluminum alloys}

\begin{abstract}
The use of chromates in aluminum alloys with aeronautical applications has been limited because of the high toxicity of these compounds. This has spurred intensive efforts to develop alternative effective and innocuous inhibitors. In this work, corrosion inhibition of a light and high strength $\mathrm{Al}-\mathrm{Li}-\mathrm{Cu}$ alloy (8090) is studied in $\mathrm{NaCl}$ solutions with $\mathrm{CeCl}_{3}$ and $\mathrm{LaCl}_{3}$ lanthanide salts added. The corrosion rate is reduced at least one order of magnitude with concentrations between 100-10,000 ppm. SEM, EDS and XPS techniques are used to characterize the rare earth cations incorporated into the surface as oxides and hydroxides.
\end{abstract}

Keywords Aluminum alloys. Corrosion. Inhibitors. Lanthanide salts.

\section{INTRODUCCIÓN}

Las aleaciones de Al-Li han supuesto un importante desarrollo en la búsqueda de materiales que aligeren el peso de las estructuras de los aviones, porque permiten la disminución de la densidad hasta un $10 \%$ y mantener la elevada resistencia mecánica de las aleaciones de aluminio convencionales. Además, presentan unos buenos valores de ductilidad y de resistencia al daño ${ }^{[1]}$.

Estas aleaciones deben ser protegidas con tratamientos superficiales que, en la actualidad, están basados en cromatos. Las importantes restricciones medioambientales, que derivan de la elevada toxicidad de estos compuestos, hacen necesario el reemplazo de los actuales sistemas que utilizan $\mathrm{Cr}$ (IV) ${ }^{[2}$ y 3$]$. En este sentido, las sales lantánidas del tipo $\mathrm{LnCl}_{3}$ se vienen usando, fundamentalmente, en aleaciones de aluminio, tanto como inhibidores como en el desarrollo de capas de conversión ${ }^{[4]}$.

A pesar del extenso número de trabajos recogidos en la bibliografía sobre la aplicación de estos compuestos lantánidos a aleaciones de aluminio ${ }^{[5]}$, no existen trabajos que se refieran a su aplicación en aleaciones pertenecientes a la familia de las de Al-Li.

Así pues, este trabajo relaciona el empleo de sales lantánidas de cerio y lantano como inhibidores de la corrosión de una aleación ligera de alta resistencia (8090-T8171), en el intento de buscar alternativas eficaces contra la corrosión y medioambientalmente limpias, que sustituyan a los cromatos. 


\section{MATERIALES Y PROCEDIMIENTO EXPERIMEN- TAL}

La aleación estudiada pertenece a la familia de aleaciones de Al-Li y su composición elemental (en $\%$ en masa) es la que figura en la tabla I.

La 8090 fue suministrada en estado T8171 que es un tratamiento que define una condición próxima al pico de envejecimiento.

Como medio corrosivo se empleó una disolución de $\mathrm{NaCl}$ al 3,56 \% en masa. Asimismo, para estudiar el efecto inhibidor de las sales lantánidas en la 8090 , se añadieron a dicha disolución concentraciones de 100, 250, 500, 1.000 y $10.000 \mathrm{ppm}$ de $\mathrm{CeCl}_{3} \cdot 7 \mathrm{H}_{2} \mathrm{O}$ y de $\mathrm{LaCl}_{3} \cdot 7 \mathrm{H}_{2} \mathrm{O}$.

La superficie de las muestras se examinó antes y después de la inmersión durante diferentes períodos de tiempo en los medios de ensayo, con el objetivo de identificar las fases responsables del proceso de corrosión y de inhibición mediante microscopía electrónica de barrido (SEM), espectroscopía de dispersión de energías (EDS) y espectroscopía fotoelectrónica de rayos X (XPS).

Por otra parte, se llevaron a cabo ensayos potenciodinámicos lineales después de $1 \mathrm{~h}$ de inmersión en el medio. Para ello, se empleó una de celda de tres electrodos, donde el electrodo de trabajo estaba formado por una superficie de $1 \mathrm{~cm}^{2}$ del material de estudio. Como electrodo de referencia se empleó un electrodo de calomelanos saturado (SCE) y como contraelectrodo un hilo de platino. Se calcularon los porcentajes de inhibición para todas las concentraciones de elementos lantánidos.

\section{RESULTADOS Y DISCUSIÓN}

Antes de determinar el efecto inhibidor de las sales lantánidas, se analizó el papel de los distintos constituyentes en el comportamiento frente a la corrosión de la 8090 en $\mathrm{NaCl}$.

La inspección de la superficie previa a su inmersión, permite identificar algunas de las fases características de las aleaciones Al-Li. Aunque $\delta(\mathrm{AlLi})$ y $\delta^{\prime}\left(\mathrm{Al}_{3} \mathrm{Li}\right)$ son los principales constituyentes de la 8090 , a las cuales se debe fundamentalmente su elevada resistencia mecánica, no se detectan mediante SEM, debido a su pequeño tamaño. Las fases endurecedoras $\mathrm{T}_{1}\left(\mathrm{Al}_{2} \mathrm{CuLi}\right)$ y $\mathrm{T}_{2}\left(\mathrm{Al}_{6} \mathrm{CuLi}_{3}\right)$ se localizan en frontera de grano y los dispersoides $\beta^{\prime}\left(\mathrm{Al}_{3} \mathrm{Zr}\right)$, que frenan el proceso de recristalización, se encuentran distribuidos en el interior de los granos. Además, las impurezas de hierro que forman los intermetálicos $\left(\mathrm{Al}_{7} \mathrm{Cu}_{2} \mathrm{Fe}\right)$ y $\left(\mathrm{Al}_{3} \mathrm{Fe}\right)$ están situados en frontera de grano siguiendo la dirección de laminación ${ }^{[6]}$.

Una vez caracterizada la microestructura de la aleación se procedió a su inmersión en el medio agresivo. Aunque a simple vista no es posible detectar la corrosión localizada en la superficie de la aleación hasta después de $8 \mathrm{~h}$ de ensayo, mediante microscopía electrónica es posible detectar el inicio del ataque en la AA8090 después de 1 h en $\mathrm{NaCl}$. Por ejemplo, en la figura 1 se muestra la disolución preferencial de la matriz adyacente a las inclusiones ricas en hierro por formación de un par galvánico en el que los intermetálicos poseen carácter catódico.

Con el aumento del tiempo de inmersión, las picaduras nucleadas durante las primeras horas de ensayo, aumentan en número y crecen en extensión. En numerosas zonas se revela el ataque de las fronteras de grano o subgrano en las que se aprecian los huecos dejados por la disolución de las fases $\mathrm{T}\left(\mathrm{T}_{1}\right.$ ó $\left.\mathrm{T}_{2}\right)$ que tienen carácter anódico. $\mathrm{Al}$ mismo tiempo, los $\mathrm{Al}_{3} \mathrm{Fe}$ han desaparecido de la superficie por su descolgamiento de la matriz con el avance de la disolución.

La adición de $\mathrm{CeCl}_{3}$ y $\mathrm{LaCl}_{3}$ al medio con cloruros provoca una importante mejora en el estado superficial de la aleación 8090 , desde el punto de vista de la corrosión localizada, no sólo durante las primeras horas de ensayo sino, también, después de 1 mes de inmersión. Esto, se debe a que la adición de dichas sales al medio provoca la formación de una capa que posee características protectoras.

Esta película comienza a formarse desde los primeros momentos de inmersión y se caracteriza por la existencia de unas partículas aisladas de varias micras de tamaño y con diferentes morfologías, que son ricas en los elementos lantánidos (Ce y La) y

Tabla I. Composición elemental (\% masa) de la aleación Al-Li-Cu (8090)

Table I. Elemental composition of Al-Li-Cu (8090) alloy (wt. \%)

\begin{tabular}{|c|c|c|c|c|c|c|c|c|c|}
\hline & Al & $\mathrm{Li}$ & $\mathrm{Cu}$ & $\mathrm{Mg}$ & $\mathrm{Zr}$ & $\mathrm{Fe}$ & $\mathrm{Si}$ & $\mathrm{Na}$ & Otros \\
\hline 8090 & bal. & 2,40 & 1,15 & 0,67 & 0,11 & 0,05 & 0,02 & 0,0004 & 0,07 \\
\hline
\end{tabular}



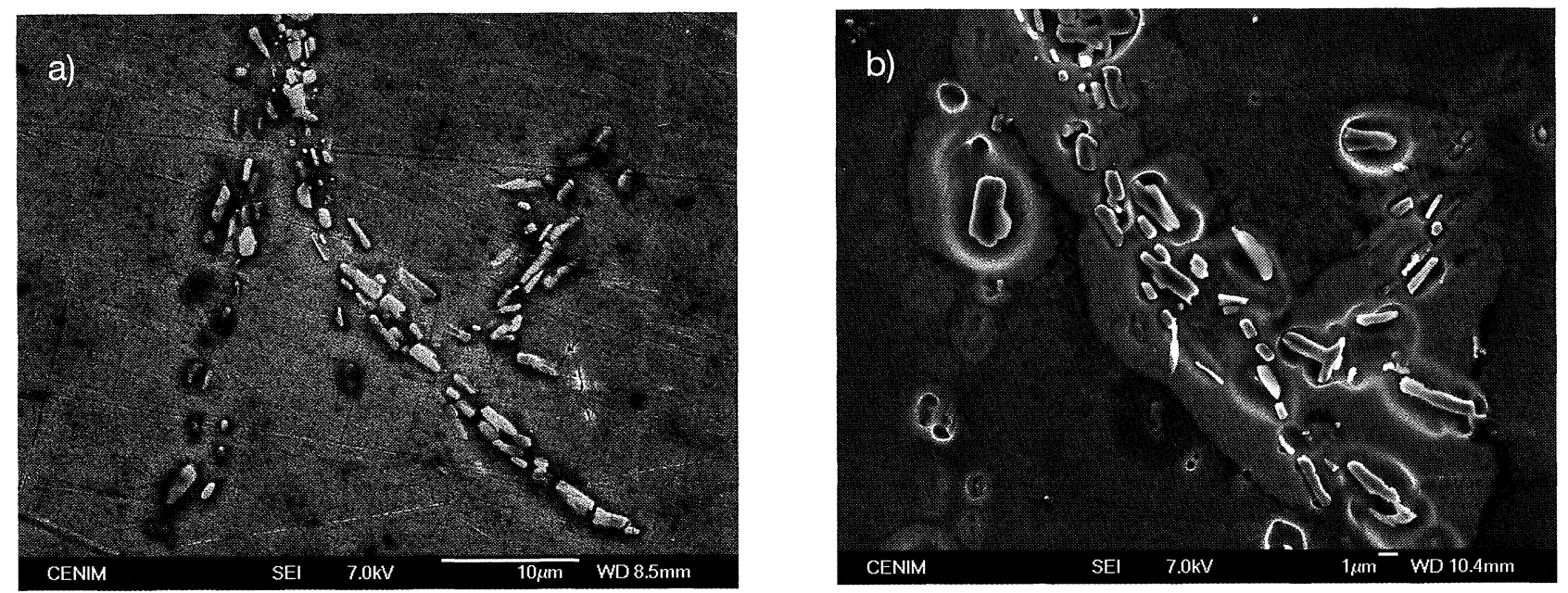

Figura 1. Micrografías de SEM que revelan el ataque localizado alrededor de los intermetálicos ricos en hierro: a) antes de su inmersión y b) después de $1 \mathrm{~h}$ en $\mathrm{NaCl} 3,56 \%$.

Figure 1. SEM micrographs of localized dissolution around iron rich intermetallics a) before immersion, b) after $1 \mathrm{~h}$ in $\mathrm{NaCl}$ $3,56 \%$

oxígeno (Fig. 2). Mediante XPS se ha comprobado el tipo de enlace entre estos elementos incorporados a la superficie. Los espectros de alta resolución de las regiones Ce $3 \mathrm{~d}$ y La3d muestran la señal típica correspondiente a las especies $\mathrm{Ce}^{3+}$ y $\mathrm{La}^{3+}$, respectivamente (Fig. 3) ${ }^{[7]}$. Por otra parte, la deconvolución de los espectros del O1s para los ensayos con inhibidor, revelan una triple contribución a la señal atribuible al óxido de aluminio y a los enlaces $\mathrm{Ln}^{3+}-\mathrm{O}$ y $\mathrm{Ln}^{3+}-\mathrm{OH}^{-}$, donde $\mathrm{Ln}^{3+}$ corresponde al $\mathrm{Ce}^{3+}$ y al $\mathrm{La}^{3+}$, en cada caso ${ }^{[8]}$. Estos resultados indican que la película generada sobre la superficie de la aleación está compuesta por óxidos e hidróxidos de $\mathrm{Ce}^{3+} \mathrm{O} \mathrm{La}^{3+}$ y óxido de aluminio.
Es posible comparar la eficacia del cerio y del lantano para las diferentes concentraciones de inhibidor en el medio con los datos obtenidos de las curvas de polarización. En la figura 4 se muestra la comparación de las curvas correspondientes a las dos concentraciones de inhibidor que ofrecen el mejor comportamiento frente a la corrosión, evaluado anteriormente en los ensayos de inmersión.

Se puede comprobar, que mientras el proceso en $\mathrm{NaCl}$ está controlado por la difusión del oxígeno con una densidad de corriente límite de difusión de $10^{-6} \mathrm{~A} / \mathrm{cm}^{2}$, la existencia de cerio o lantano en el medio provoca el descenso en el potencial de corrosión del sistema en más de $200 \mathrm{mV}$ y de la

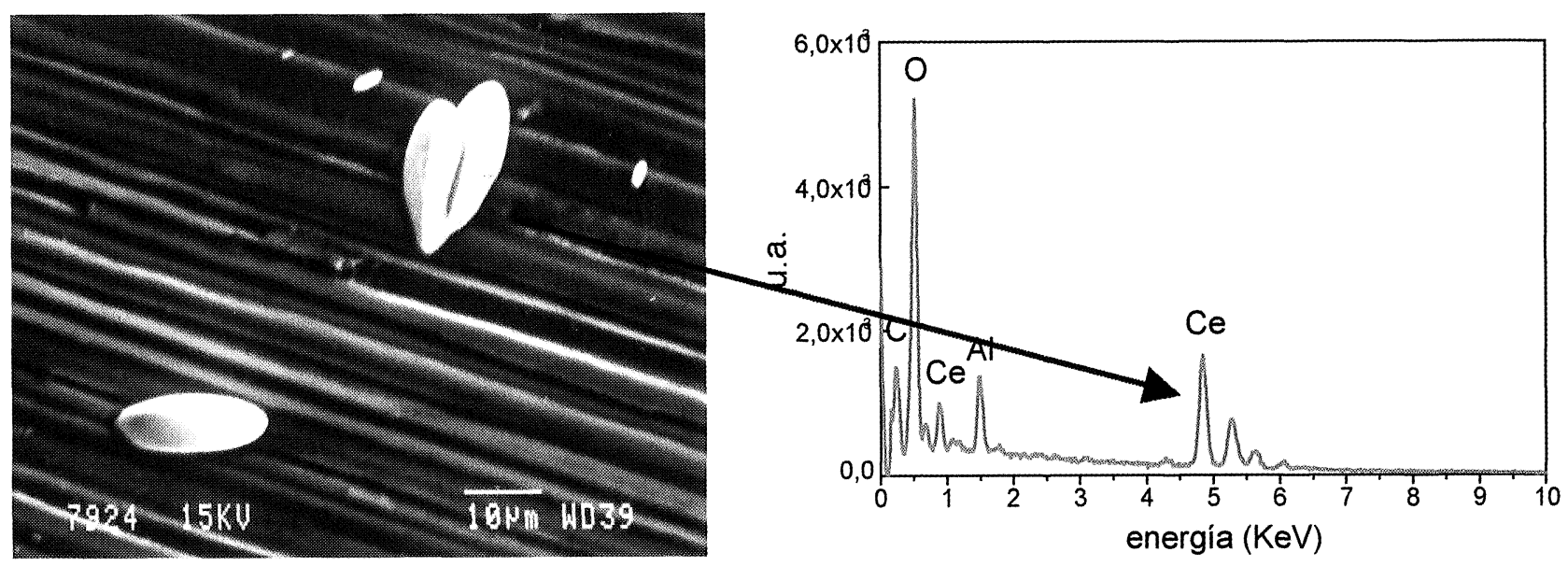

Figura 2. Micrografía de SEM que muestra el aspecto de la superficie de la aleación 8090 después de 1 mes de inmersión en $\mathrm{NaCl} 3,56 \%+1.000 \mathrm{ppm}$ de $\mathrm{CeCl}_{3} \cdot 7 \mathrm{H}_{2} \mathrm{O}$. El EDS refleja la composición de una de las partículas rica en $\mathrm{Ce}$ y $\mathrm{O}$.

Figure 2. SEM micrograph showing the appearance of the surface of 8090 alloy after 1 month of immersion in $\mathrm{NaCl} 3,56 \%+$ $1,000 \mathrm{ppm}$ de $\mathrm{CeCl}_{3}$. $7 \mathrm{H}_{2} \mathrm{O}$. EDX analysis reveals the composition in $\mathrm{Ce}$ and $\mathrm{O}$ of some particles. 

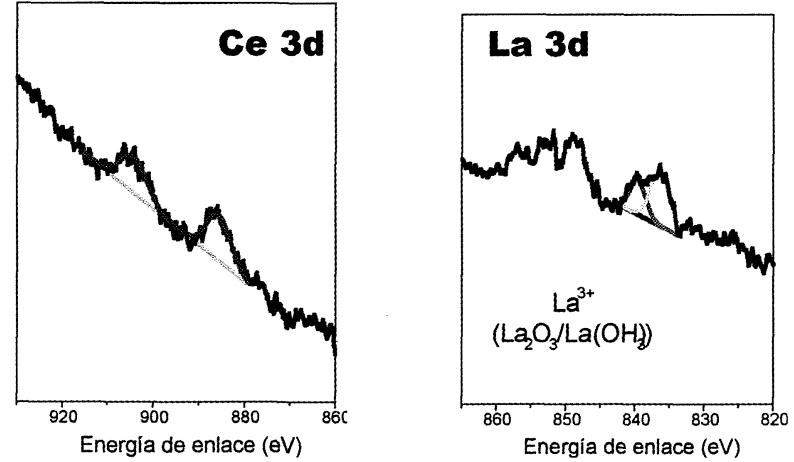

Figura 3. Espectros de XPS del Ce 3d y La 3d que confirman la existencia de los cationes lantánidos en la superficie de la aleación 8090.

Figure 3. XPS spectrum of Ce3d and La3d confirming the existence of lanthanide cations on the surface of 8090 alloy.

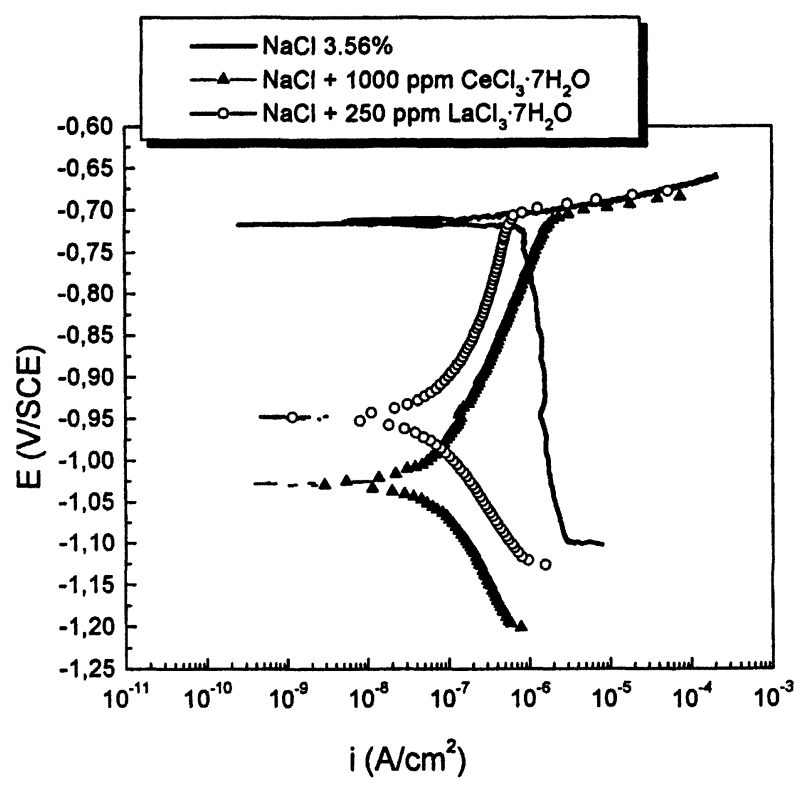

Figura 4. Comparación de las curvas de polarización potenciodinámica de la AA8090 después de $1 \mathrm{~h}$ de inmersión en $\mathrm{NaCl} 3,56 \%, \mathrm{NaCl} 3,56 \%+1.000$ ppm de $\mathrm{CeCl}_{3}$ y $\mathrm{NaCl} 3,56 \%+250 \mathrm{ppm} \mathrm{LaCl}_{3}$.

Figure 4. Comparison of potenciodynamic polarization curves of AA8090 after $1 \mathrm{~h}$ of immersion in $\mathrm{NaCl} 3,56 \%$, $\mathrm{NaCl} 3,56 \%+1,000 \mathrm{ppm}$ de $\mathrm{CeCl}_{3}$ y $\mathrm{NaCl} 3,56 \%+$ 250 ppm $\mathrm{LaCl}_{3}$.

densidad de corrosión en, al menos, un orden de magnitud $\left(10^{-7}-10^{-8} \mathrm{~A} / \mathrm{cm}^{2}\right)$. Estos resultados indican un mecanismo de inhibición catódico, en el que los óxidos e hidróxidos de los cationes lantánidos precipitan en las áreas catódicas y frenan la velocidad de la reacción de corrosión.

En la figura 5 se compara la eficacia inhibidora obtenida para todas las concentraciones de sa-

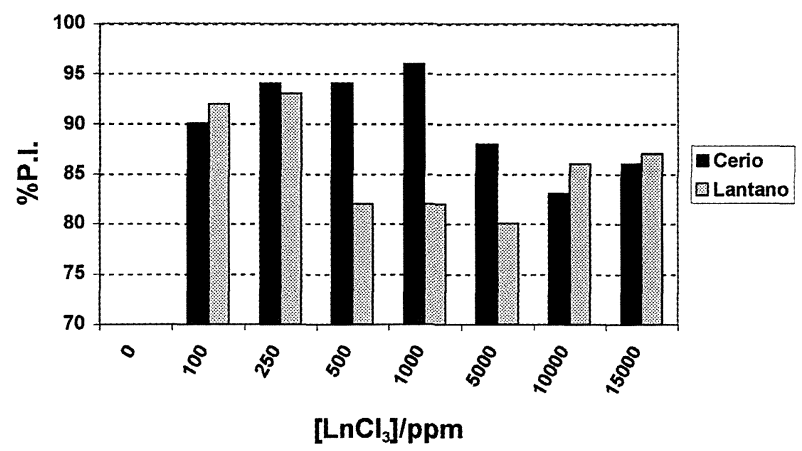

Figura 5. Porcentajes de inhibición obtenidos para la aleación AA8090 con todas las concentraciones de cerio y lantano ensayadas después de $1 \mathrm{~h}$ de inmersión.

Figure 5. Inhibition percentages obtained with every used cerium and lanthanum concentrations for AA8090 alloy after $1 \mathrm{~h}$ of test.

les lantánidas. El mayor porcentaje de inhibición (96\%) se alcanza con 1.000 ppm de cerio, mientras que con lantano se consigue, como máximo, un $93 \%$ con 250 ppm. Además, el rango de concentraciones para el que el \% P.I. supere un $90 \%$ es mayor en el caso del cerio que en el lantano.

Aún no se sabe con certeza la razón de este comportamiento diferente entre cerio y lantano y de que grandes concentraciones de inhibidor supongan un efecto contraproducente en la efectividad de la inhibición. En este sentido, se ha relacionado la efectividad de las sales lantánidas con dos factores:

- La naturaleza de la capa protectora: se ha observado que, a partir de 1.000 ppm de catión lantánido, las películas de óxidos son más pulverulentas y defectuosas ya que, por ejemplo, con 10.000 ppm de $\mathrm{CeCl}_{3}$, después de 1 mes de inmersión, se forma una capa amarilla pulverulenta muy poco adherente, que al microscopio electrónico muestra por una parte menor cantidad de partículas grandes ricas en cerio, y por otra, mayor degradación de la superficie, con numerosos productos de corrosión y picaduras.

- El pH del medio: la formación de la capa se produce mediante la hidrólisis de los cationes lantánidos debido al aumento local del $\mathrm{pH}$ en las zonas catódicas de la superficie. Sin embargo, para un $\mathrm{pH}$ determinado, según los diagramas de Pourbaix, se necesita mayor concentración de lantano para que precipite $\mathrm{La}(\mathrm{OH})_{3}$ que de cerio para que precipite $\mathrm{Ce}(\mathrm{OH})_{3}{ }^{[9]}$. Por ello, parece probable que cuando ocurre un aumento de basicidad, la precipitación del 
hidróxido de cerio está más favorecida que la del hidróxido de lantano, lo cual podría explicar las pequeñas diferencias existentes en la capacidad inhibidora de ambos.

\section{CONCLUSIONES}

La adición de $\mathrm{CeCl}_{3}$ o $\mathrm{LaCl}_{3}$ en concentraciones a partir de $100 \mathrm{ppm}$ al $\mathrm{NaCl}$ disminuye la cinética de corrosión de la aleación ligera de $\mathrm{Al}-\mathrm{Li}-\mathrm{Cu}$ 8090 hasta largos períodos de inmersión. La inhibición se produce por la formación de una película de óxidos e hidróxidos del catión lantánido que bloquea las zonas catódicas y frena la reacción global. Se consiguen mejores porcentajes de inhibición con cerio que con lantano en un rango más amplio de concentraciones.

\section{REFERENCIAS}

[1] A.A. GokHale y T.R. RamachandRan, Indian J. Technol. 28 (1990) 235-246.

[2] G. Bierwogen, J. Coat. Technol. 73 (2001) 45-52.

[3] W.G. Fahrenholtz, M.J. O’Kefe, H. Zhou y J.T. Grant, Surf. Coat. Technol. 155 (2002) 208-213.

[4] B.R.W. Hinton, Corrosion/89 symposium, A. Raman y P. Labine (eds.), NACE, paper no I-11. pp. 1-19.

[5] A. Aballe, M. Bethencourt, FJ. Botana, M. Marcos, J. Pérez y M.A. Rodríguez, Rev. Metal. Madrid 33 (1997) 363-369.

[6] R. Ambat y E.S. Dwarakadasa, Br. Corros. J. 28 (1993).

[7] D.R. ARnotT y N.E. Ryan, Applications Surf. Sci. 22-23 (1985) 236-251.

[8] M. Dabalà, L. Armelao, A. Buchberger e I. Calliari, Appl. Surf. Sci. 172 (2001) 312-322.

[9] Atlas of Electrochemical Equilibria in Aqueous Solutions, $1^{\text {st }}$ Ed., Pergamon Press, London, 1966, pp. 183-197. 